\title{
Large bugs damage pistachio nuts most severely during midseason
}

\author{
Kent M. Daane \\ Glenn Y. Yokota \\ Rodrigo Krugner \\ Shawn A. Steffan \\ Paul G. da Silva \\ Robert H. Beede \\ Walter J. Bentley \\ Gary B. Weinberger \\ $\nabla$
}

"Large bug" damage to pistachio nuts varies by season, as well as among insect species and development stages, with larger bugs typically causing more damage than smaller bugs. We investigated pistachio damage by three large bug species (leaffooted bug, redshouldered stink bug and flat green stink bug) at different development stages and throughout the season, using field surveys and cage studies. Before fruit set occurs in June, most damaged nuts are dropped from the cluster without reducing fruit load. The midseason period (June to July) is the most critical because the damaged nuts remain in the cluster. After shell hardening, the kernel is largely protected from bug feeding.

$\neg$ hough once considered virtually 1 pest-free, California pistachios are now attacked by a variety of insects, especially the Hemiptera or true bugs. Most are native pests that built resident populations as pistachio orchard acreage increased. These pests are commonly grouped as "small" and "large" bugs. The small bugs (adults about onequarter inch) include several species of Miridae and Rhopalidae, most importantly Calocoris norvegicus (Gmelin), Phytocoris relativus (Knight) and Lygus hesperus (Knight). The large bugs (adults about one-half inch) include some species of leaffooted bugs (Coreidae), such as Leptoglossus clypealis (Heidemann); and stink bugs (Pentatomidae), such as the redshouldered stink bug (Thyanta pallidovirens [Stål]), the flat green stink

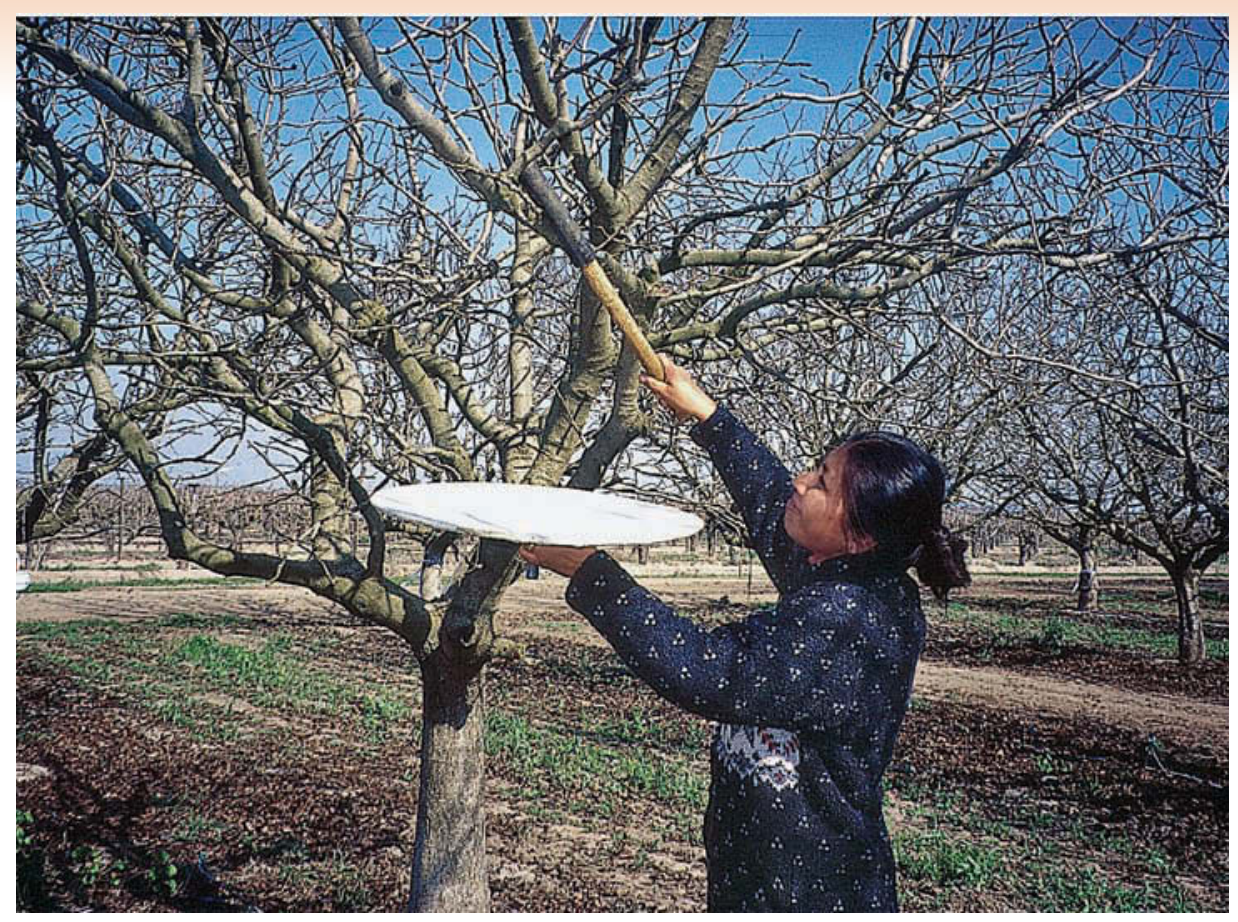

Raksha Malakar-Kuenen, a postdoctoral researcher, whacks a dormant pistachio limb with a mallet to dislodge overwintering stink bugs onto a beating tray, one of the primary field monitoring methods. California pistachios, once considered virtually pest free, are now under attack from a variety of insects, especially "true" bugs of the Hemiptera order.

Significant crop loss will occur only when early-season bug densities are so high that the number of dropped nuts exceeds the level that the tree will naturally compensate.

bug (Acrosternum hilare [Say]), Uhler's stink bug and Chlorochroa uhleri (Stål) species. All of these bugs use piercingsucking mouthparts to feed directly on the nut. While small bugs are often abundant and can cause damage early in the season, they pose little threat after shell hardening (Michailides et al. 1987; Purcell and Welter 1991). Large bugs, on the other hand, have larger, stronger mouthparts and may continue to damage kernels until harvest time (Michailides et al. 1987).

There are also seasonal differences in the type of feeding damage that oc- curs. Early in the season, when the nuts are small (less than 10 millimeters in diameter), feeding punctures through the fruit pericarp (the outer wall of the nut, comprised of the outer epicarp, the fleshy middle mesocarp and the inner endocarp) quickly result in darkened areas of the hull or epicarp lesions (Uyemoto et al. 1986; Bostock et al. 1987), and the damaged nuts often drop from the cluster (Purcell and Welter 1991). In midseason, fruit load is set but shell lignification (hardening) is not complete. The damaged nut remains in the cluster and the resulting epicarp lesion can stain the outer shell, lowering market value. During this time, the kernel is also developing and feeding punctures can result in necrotic spots on the nut meat (kernel necrosis), aborted nuts or fungal contamination (such as stigmatomycosis).

Therefore, both the bug species present and the seasonal period will affect pest control decisions. We investigated pistachio damage by three large bug species (leaffooted bug, redshouldered 
Monitoring pistachio orchards is essential to determine the type of insect pest present and the nature of potential damage. Midseason is the most critical period, but damage can occur early and late in the season as well. The insects in this guide are among the most common.

\section{LARGE BUGS}

Large bugs (adults approximately $1 / 2$ inch $\longmapsto$ ) have larger, stronger mouthparts and may continue to damage kernels until harvest.

\section{Redshouldered stink bug}

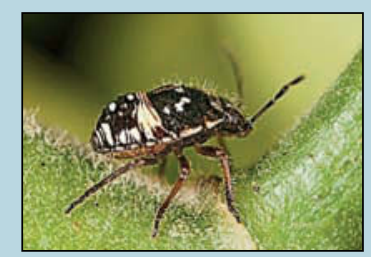

Small nymph

Flat green stink bug

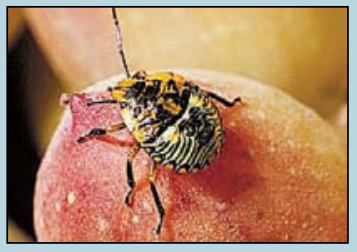

Small nymph

Leaffooted bug

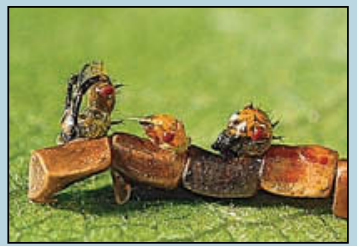

Nymphs emerging

from egg mass

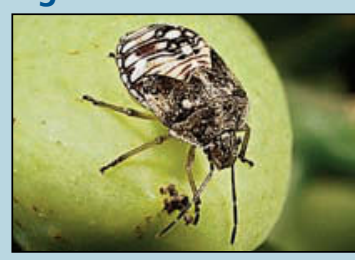

Large nymph

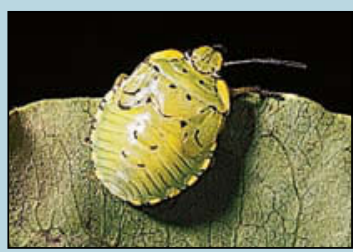

Large nymph

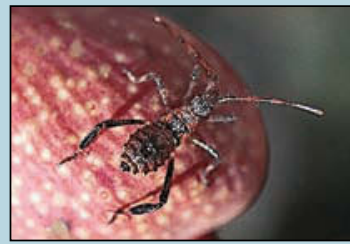

Medium nymph**

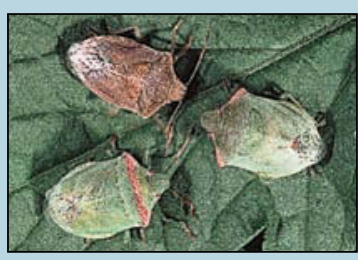

Adult*

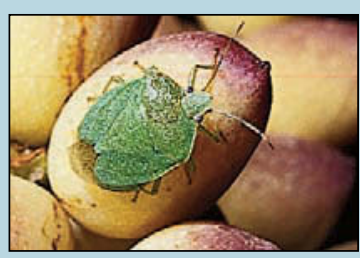

Adult

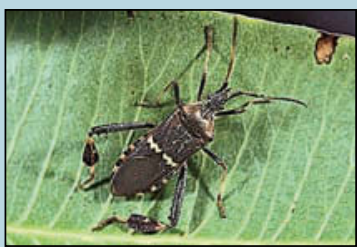

Adult**

\section{SMALL BUGS}

Small bugs (adults approximately $1 / 4$ inch $\longmapsto$ ), sometimes called "plant bugs," are often more abundant and can cause damage early in the season. Adults pose little threat after shell hardening.

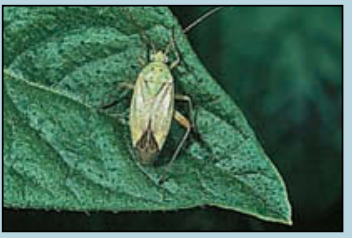

Calocoris (adult)

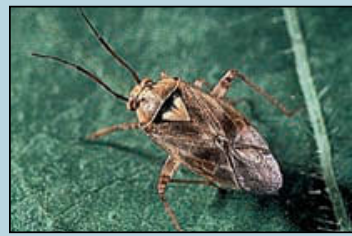

Lygus bug (adult)

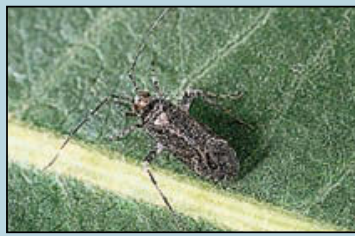

Phytocoris (adult) stink bug and flat green stink bug) at different development stages and throughout the season. Our work can improve pest control decisions by describing seasonal periods when large bug feeding results in greater crop loss.

\section{Pistachio orchard studies}

Field surveys. To compare seasonal changes in nut damage with changes in pest species, we sampled nine orchards in the San Joaquin Valley in 1998. To sample bugs in the pistachio trees, we took 350 beating-tray samples per orchard every 2 to 3 weeks from April to November. For a beating tray sample, a cloth tray is held under a section of the pistachio tree, and when the branch above is struck with a mallet, the dislodged insects are caught on the tray. To sample bugs in the cover crops, we took 350 sweep-net samples per orchard every 1 to 2 weeks from March to September. For this, a cloth insect net was swept through the cover crop, using 50 continuous sweeps while walking through the cover crop for each bag of collected insects (seven sections of each orchard were sampled). Feeding damage was determined by recording the number of epicarp lesions in 700 pistachio nut clusters per orchard every 7 to 14 days from May to September.

Cage studies - seasonal damage. In 1998, we conducted cage trials at the UC Kearney Research and Extension Center. After bud-break (when plant dormancy ends), pistachio branches with nut clusters were isolated in 3-gallon (11.4-liter) organdy cages. The number of nuts per cluster was recorded, and then a single bug was introduced for a 7-day feeding period. After that, the bugs were removed and the numbers of total, dropped and damaged (epicarp lesion) nuts were recorded. The cages were then resealed until harvest time, when the numbers of total, dropped and damaged nuts were again recorded. The nuts were then dissected and the kernel condition (clean, necrotic, stigmatomycosisaffected or aborted) was recorded.

The trials were conducted at three seasonal periods that correspond to changes in fruit susceptibility to bug damage: early season, as the fruit load is being set (May 1 to 8); midseason, dur- 


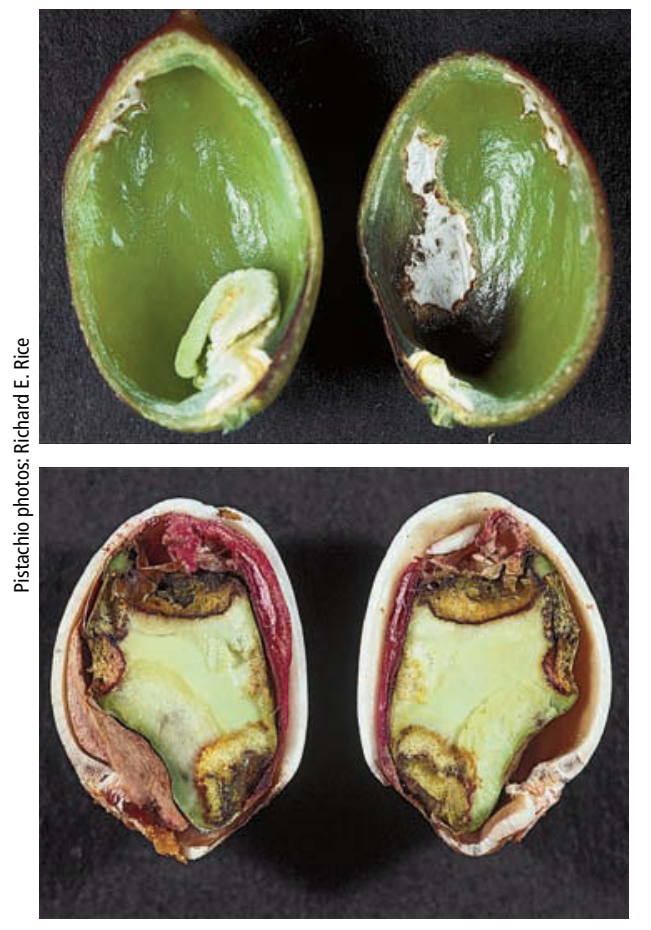

ing shell hardening (June 10 to 17); and late season, after shell hardening (Aug. 13 to 21). We included the small bug Calocoris in some of the early trials as a comparable marker to previously published studies. The early-season treatments were adult redshouldered stink bug (RSSB), flat green stink bug (FGSB) and Calocoris. The midseason treatments were adult redshouldered stink bug, flat green stink bug and Calocoris, and the small (second or third instars) and large (fourth or fifth instars) stages of redshouldered stink bug and flat green stink bug. The late-season treatments were adult redshouldered stink bug, flat green stink bug and leaffooted bug, and small (second or third instars) and large (fourth or fifth instars) stages of redshouldered stink bug and flat green stink bug. We also did a no-insect control treatment. For each trial, treatments were set in a complete randomized block design, with five replicates (cages) per treatment (130 cages total). Blocks were individual or adjacent trees.

Cage studies - "hidden" damage. We call damage after shell hardening is complete "hidden," because epicarp lesions will not form even though some of the bug's probes can successfully penetrate the shell to feed on the kernel. In 2001, we further quantified bug damage after the midseason period by inoculating clusters with different bug species every few weeks. Pistachio

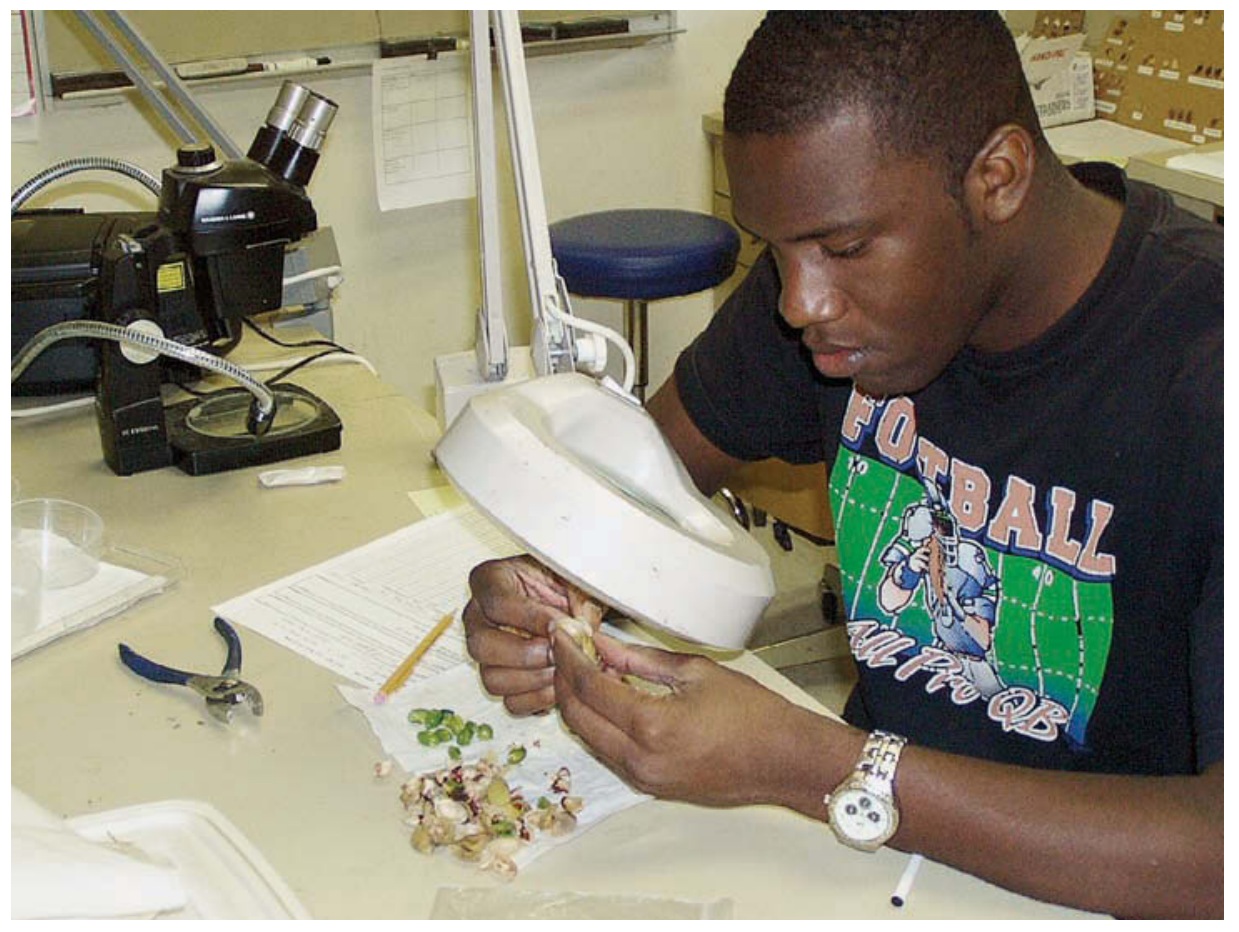

Above right, Gary Guelce, a research technician at KREC, examines pistachio kernels for evidence of damage. The most critical period for potential kernel damage occurs midseason, when large bug mouthparts can easily penetrate hardening shells. Damaged kernels may not become apparent until harvest. However, generalizing about bugs and nut damage is difficult because of regional and seasonal variations. Damage to the interior of the pistachio nut can range from blanks, top left, which can be caused by large or small bugs early in the season, to late season necrosis, bottom left, caused primarily by the larger bugs.

Statistical analysis. The results are presented as means per treatment ( \pm SEM) and compared using analysis of variance (ANOVA), with treatment means separated with Tukey's HSD test. When needed, data were transformed $(\sqrt{x})$ to normalize the variance. In the cage study of hidden damage, levels of kernel necrosis in each bug treatment were adjusted to the control (Abbott 1925).

\section{Bugs found in field surveys}

Overall, insect collections using the beating tray were low (less than 0.2 bugs per sample). In the surveyed orchards, Phytocoris was the most abundant small bug in the canopy, with three seasonal peaks in late April, mid-June and late August. Flat green stink bug was the most common large bug in the canopy and was found from mid-May 


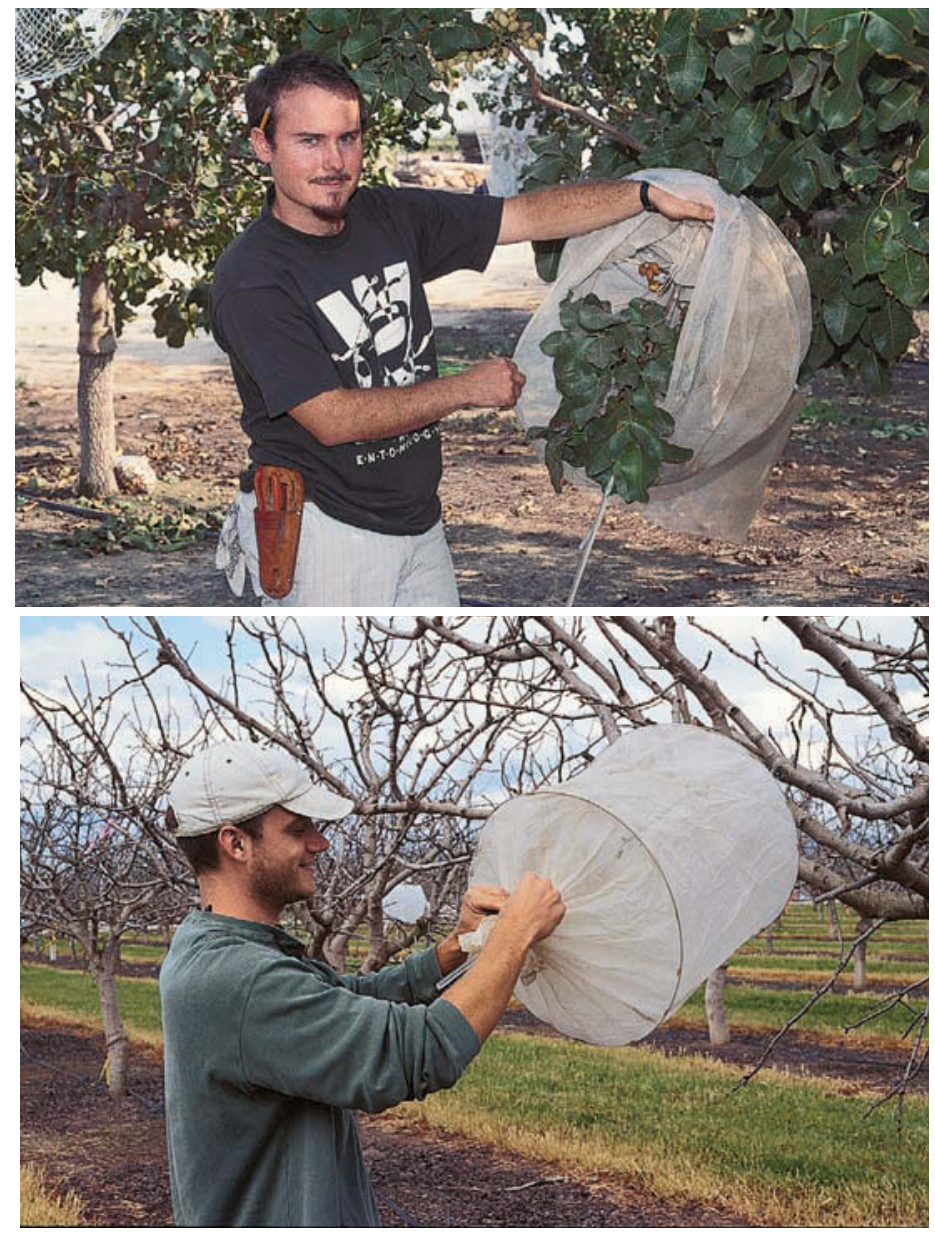

Field studies of pistachio nut damage and drop included a stink bug feeding trial. Bottom, former UC lab assistant Cody Anderson ties off a 10-gallon organdy cage during dormancy to isolate pistachio clusters prior to insect damage. Top, former UC staff research associate and study co-author Shawn Steffan opens one of the cages to inspect midseason nut condition.

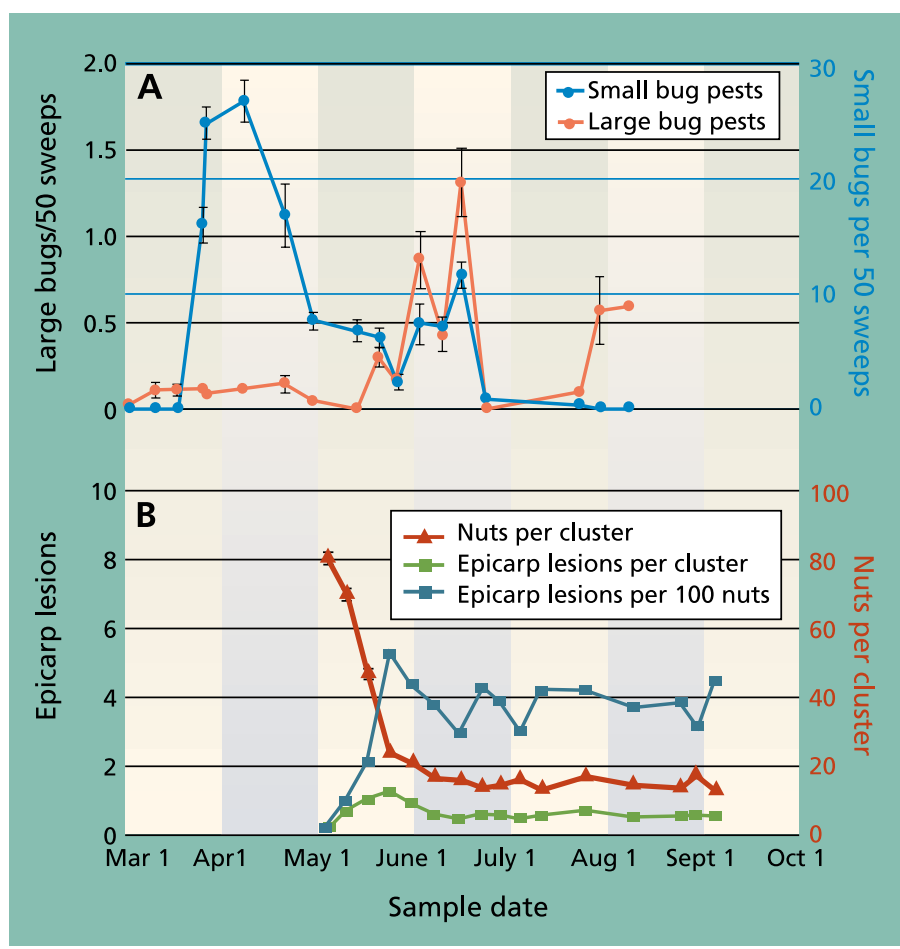

Fig 1. (A) Average ( \pm SEM) number of small and large bugs collected from cover crops in nine sampled orchards in the San Joaquin Valley shows that small bugs, primarily Calocoris, were most common early in the season, while large bugs, most commonly stink bugs, were most common in June. (B) Average ( \pm SEM) total and damaged nuts per cluster in the sampled orchards. until harvest. Flat green stink bug commonly overwinters in the pistachio orchard and is often the first large bug found each year. We also collected redshouldered stink bug from July through harvest. There were no leaffooted bugs collected in the beating tray samples; however, we observed these bugs in the canopy from July through harvest.

From sweep-net samples of the ground covers, we identified 29,873 specimens of bug pests. Most bugs were collected in spring (fig. 1A), and were composed primarily of Calocoris $(63 \%)$ and Lygus (21\%). Large bugs were less common, collected most often in June, and composed primarily of redshouldered stink bug and flat green stink bug. The data on the seasonal changes in bug species in monitored orchards is presented in the following discussion on nut damage. However, it is difficult to generalize about bugs and nut damage because of seasonal and regional varia- tions in small and large bug species. For example, while Neurocolpus longirostris (Knight) may be one of the more damaging bugs, it is also rare; and while Uhler's stink bug may be common in orchards on the West Side of the San Joaquin Valley, the redshouldered stink bug and flat green stink bug are more dominant on the East Side.

\section{Nut drop and damage}

When the nut cluster is formed in April, it has more nuts than it will carry to harvest. Therefore, the large number of dropped nuts per cluster observed in May resulted primarily from a natural drop as fruit load was set (fig. 1B). Nevertheless, there was also an impact from insect feeding. We observed Phytocoris and Calocoris feeding on newly formed nuts in April and most of these damaged nuts dropped within 24 hours, without any visible signs of insect damage. This is because it takes more than 24 hours for visibly darkened epicarp lesions to form (Bostock et al. 1987). The result is that insect feeding on nuts in their earliest development period is easily overlooked. Later, in late April and early May as the fruit load establishes, insect-damaged nuts (epicarp lesions) typically remain in the cluster for a short time. This visible sign of insect feeding can be used to monitor bug activity.

The number of visible epicarp lesions increased in late May and then declined, leveling off to about four per 100 nuts (less than one per cluster) in mid-June (fig. 1B). In July and August, there was essentially no change in the number of lesions (fig. 1B), even though there was an increase in the number of large bugs (fig. 1A). This is because insect wounds no longer cause epicarp lesions during fruit maturation and shell lignification (hardening), when peroxidase activity in the pericarp declines (Bostock et al. 
1987). Therefore, most lesions found after mid-July were the result of earlier feeding wounds. We suggest that the subsequent feeding by large bugs presents potential "hidden" damage, since kernel necrosis may occur without the external sign (epicarp lesion) of insect feeding. Therefore, epicarp lesions cannot be used to monitor insect activity or damage after shell hardening.

The relationship between epicarp lesions in pistachio nuts and insect feeding was first reported for the leaffooted bug by Bolkan et al. (1984), and was further clarified for large bugs by Rice et al. (1985) and for small bugs by Uyemoto et al. (1986). Michailides et al. (1987) categorized the seasonal succession of bug species in a Sacramento County pistachio orchard and compared damage levels among bug species. The results from our field-sampling build upon these studies and show that the earlyseason feeding by small and large bugs is minimized because the damaged and dropped nuts will typically not affect fruit load. This results from early-season plant compensation of fruit load (fewer nuts are dropped naturally) in response to insect damage, which was first verified with Phytocoris (Beede et al. 1996). We also confirmed that while large bugs may be present in the orchard after shell hardening is complete, there will not be an increase in epicarp lesions, as first detailed by Bostock et al. (1987).

\section{Size and species affect damage}

Initial damage. After the 7-day feeding period in the cage studies, the numbers of dropped nuts in the earlyseason trial were higher in all insect treatments other than the control (fig. 2). In the midseason trial, only the adult redshouldered stink bug and adult and large nymph flat green stink bug treatments were significantly higher than the control, and there were no treatment differences in the late-season trial (fig. 2). The pattern of dropped nuts among treatments in the midseason trial also suggests that bug size and species affect damage levels. In both

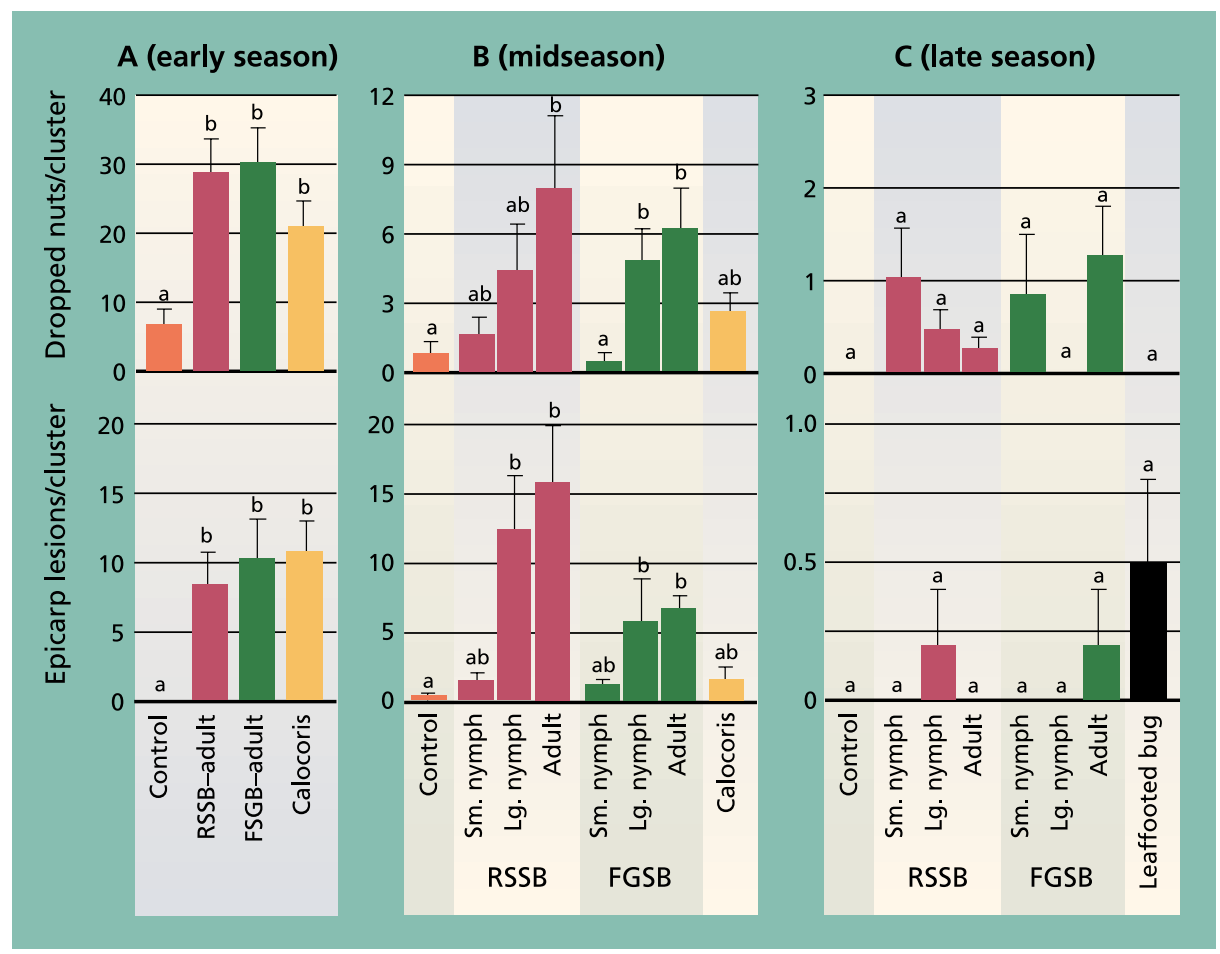

Fig. 2. Average ( \pm SEM) number of epicarp lesions and dropped nuts after a 7-day exposure period to different bug species (RSSB = redshouldered stink bug, FGSB = flat green stink bug) or development stages, in (A) early season (May 1-8), (B) midseason, during shell hardening (June 10-17) and (C) late season, after shell hardening (Aug. 13-21). In each graph, different letters above each bar indicate a significant difference between treatments (Tukey's HSD test, $P<0.05$ ).

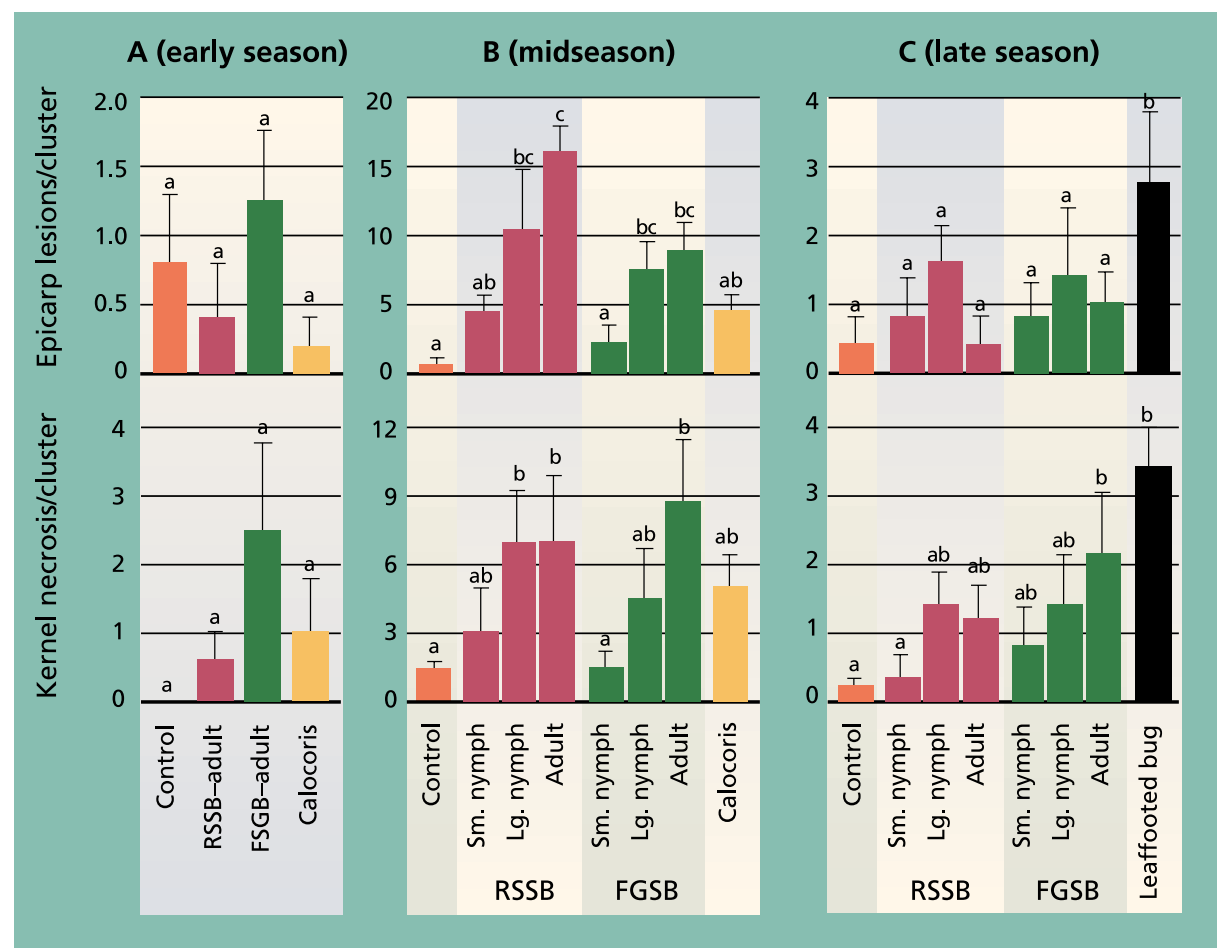

Fig. 3. Average ( \pm SEM) levels of epicarp lesions and internal nut damage (kernel necrosis) at harvest time for different bug treatments (RSSB = redshouldered stink bug, FGSB = flat green stink bug) during (A) early season (May 1-8), (B) midseason, during shell hardening (June 10-17) and (C) late season, after shell hardening (Aug. 13-21). In each graph, different letters above each bar indicate a significant difference between treatments (Tukey's HSD test, $P<0.05$ ). One replicate (cage) of the midseason Calocoris treatment was dropped from the analysis as an outlier. 


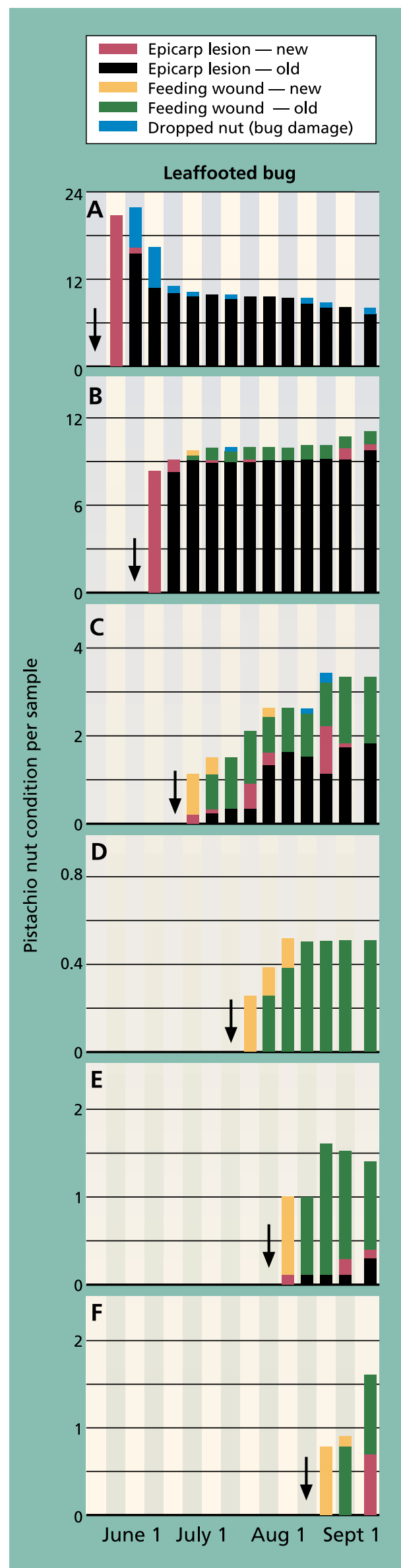

Fig. 4. Amount and type of external nut damage from a leaffooted bug exposed for a 7-day feeding period at mid- and late-season periods (A-F). Arrows indicate the date when the cages were inoculated. Each stacked bar represents averages for the number of nuts that showed the damage sign.

the redshouldered stink bug and flat green stink bug treatments, dropped nuts increased from the small nymph (and Calocoris) to adult stages. Adult redshouldered stink bug and flat green stink bug were tested in each trial period, and we used changes in these insects' damage levels to compare seasonal changes. For both the adult redshouldered stink bug and flat green stink bug, significantly more insectdamaged nuts were dropped in the earlythan in the midseason trial, and in the mid- than in the late-season trial $(\mathrm{F}=14.80, \mathrm{df}=2,12, P=0.001 ; \mathrm{F}=26.00$, $\mathrm{df}=2,12, P<0.001$; respectively).

The number of epicarp lesions formed in the early-season trial was significantly greater in all insect treatments than the control, but not different among tested insect species (fig. 2). In the midseason trial, bug species and size had an impact on the formation of epicarp lesions. As with the number of dropped nuts, there were more epicarp lesions in treatments with larger insects, with the redshouldered stink bug large nymph and adult treatments significantly higher than the control. In the late-season trial there were no treatment differences. As with nut drop, differences in the adult redshouldered stink bug and flat green stink bug treatments were used to compare seasonal periods. We found a significant reduction in epicarp lesions for both adult redshouldered stink bug and flat green stink bug in the late-season trial compared to the early- and midseason trials $(\mathrm{F}=7.189, \mathrm{df}$ $=2,12, P=0.009 ; \mathrm{F}=8.80, \mathrm{df}=2,11, P=$ 0.005 ; respectively).

Crop damage. At harvest time the impact of hemipteran feeding on crop damage is more evident. Across the trial periods, adult redshouldered stink bug and flat green stink bug feeding resulted in significantly more epicarp lesions at harvest time in the midseason trial than in either the early-season (in which damaged nuts are dropped) or late-season trial (in which epicarp lesions are not readily formed $)(\mathrm{F}=60.54, \mathrm{df}=2,12, P$ $<0.001 ; \mathrm{F}=12.99, \mathrm{df}=2,12, P<0.001$; respectively).

In the early-season trial, the number of epicarp lesions at harvest time was quite low (less than 1.5 per cluster) and was similar across all treatments (fig. 3). Most damaged nuts dropped from the cluster, as was evident from collected nuts in the cage bottom with feeding puncture wounds or epicarp lesions. Combined with data on dropped nuts (fig. 2), the results suggest that most insect-damaged nuts in the early-season period do not remain in the cluster, verifying there is crop compensation for early-season damage by redshouldered stink bug, flat green stink bug and Calocoris. In contrast, in the midseason treatment differences in epicarp lesions at harvest time were pronounced (fig. 3), with more damage associated with the larger insects. In the late-season trial, there were no treatment differences in the number of epicarp lesions in the stink bugs tested, while there was a significant difference in the leaffooted bug treatment. The few lesions found may have been the result of other pathogens that contaminated the insect feeding wounds.

The most critical measure of insect crop damage in pistachio nuts is kernel necrosis. In the early-season trial, the levels of kernel necrosis were low and not different among treatments, again a result of damaged nuts dropping from the cluster (fig. 3). In the mid- and lateseason trials, there was more damage in treatments with larger insects, as previously described. Comparing trial periods, kernel necrosis in the redshouldered stink bug and flat green stink bug treatments was significantly higher in the midseason trial than in either the early- or late-season trials $(\mathrm{F}=11.32, \mathrm{df}$ $=2,11, P<0.001 ; \mathrm{F}=17.29, \mathrm{df}=2,11, P$ $<0.001$; respectively).

These results for the early- and midseason trials are comparable to those of previous studies (Michailides 


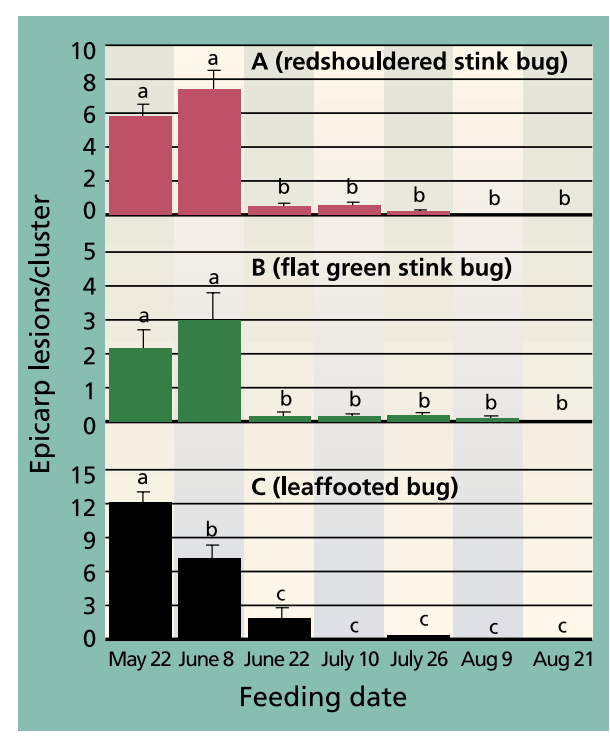

Fig. 5. Average ( \pm SEM) levels of epicarp lesions for $(A)$ redshouldered stink bug, (B) flat green stink bug and (C) leaffooted bug were significantly different among different feeding dates. For each insect, different letters above each bar indicate a significant difference between inoculation dates (Tukey's HSD test, $P<0.05$ ).

et al. 1987; Rice et al. 1985). However, the results for the late-season trial disagree with those of earlier studies on one critical point. While we found that larger bugs were better able to penetrate the hardened pistachio shell and feed on the kernel, we also found significantly less kernel damage in the late season, suggesting that the hardened shell provides some protection from even the largest bug tested, adult leaffooted bugs.

Still, there was some kernel damage, and a comparison of feeding locations provides an indication of how these large bugs are able to penetrate the pistachio shell. Michailides et al. (1988) suggest that late-season feeding by the leaffooted bug was successful when the insect's mouthparts penetrated the shell near the "Achilles heel" of the pistachio - the region near the peduncle (the nut's stem). To test this observation, we regressed the treatment means of kernel necrosis (which indicated that the bug had successfully penetrated the kernel) against epicarp lesions at the Achilles heel and epicarp lesions elsewhere. Indeed, the results confirmed that kernel necrosis was significantly and positively related to epicarp lesions at the Achilles heel $(y=3.247 x+$ $\left.0.610, \mathrm{r}^{2}=0.93, P<0.001\right)$.

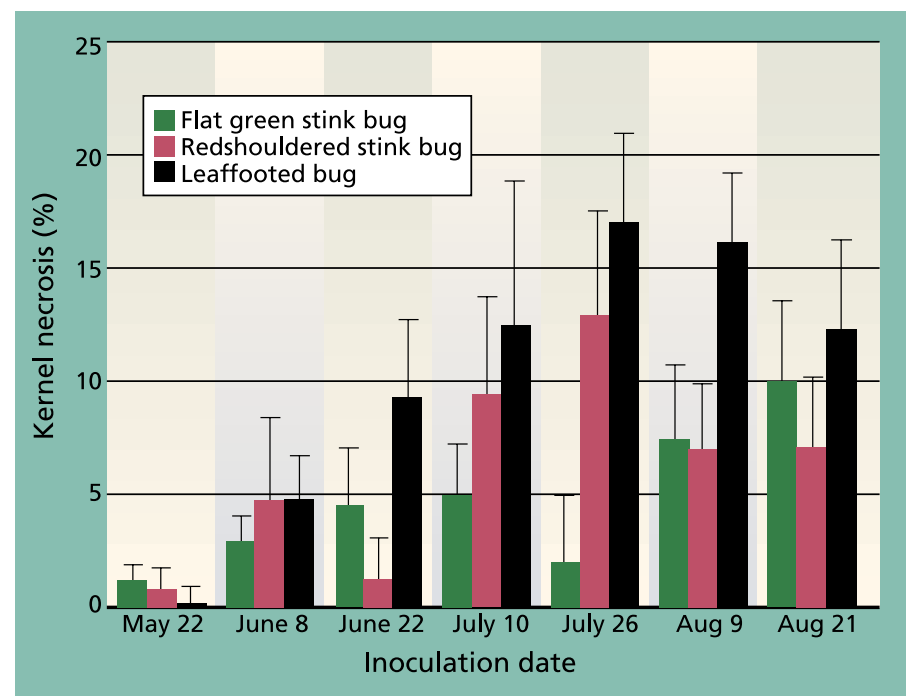

Fig. 6. Average ( \pm SEM) levels of kernel necrosis for redshouldered stink bug, flat green stink bug and leaffooted bug for different 7-day feeding periods.

\section{Hidden damage from feeding}

The experiment was designed to better understand the transition period between the visible signs of feeding damage (epicarp lesion) and "hidden" crop loss (kernel necrosis) in the mid- and late-season periods. Figure 4 shows the amount and type of external nut damage for leaffooted bug; we had similar results with redshouldered stink bug and flat green stink bug.

As with the previous cage study, the seasonal pattern of insect damage provided the most useful information. In the first trial (inoculation on May 22), insect feeding quickly resulted in new epicarp lesions. The number of new epicarp lesions per cluster was significantly different among tested insects, ranging from most to least severe in leaffooted bug $(20.7 \pm 1.7)$, redshouldered stink bug $(8.4 \pm 2.1)$ and flat green stink bug $(2.1$ $\pm 0.9)(\mathrm{F}=33.58, \mathrm{df}=2,27, P<0.001)$.

Fruit load was not complete during the May 22 inoculation period and some of the insect-damaged nuts dropped from the cluster, with significantly more nut drop per cluster in the leaffooted bug $(5.3 \pm 1.2)$ than in either redshouldered stink bug $(1.8 \pm 0.6)$ or flat green stink bug $(0.6 \pm 0.4)(\mathrm{F}=7.737, \mathrm{df}=2,27, P=$ 0.002). Most of the damaged nuts (which had visibly evident old epicarp lesions) remained in the cluster until harvest time (fig. 4A). The pattern of feeding damage in the second trial (inoculation on June 8) was similar to the first trial but had two important differences: there was now little fruit drop, and some of the new feeding wounds did not form epicarp lesions due to shell hardening (fig. 4B).

The third trial (inoculation on June 22) marks a period of considerable change in the expression of insect damage. Most important is that new and old feeding wounds did not lead to epicarp lesions for many weeks, if at all (fig. 4C). This pattern becomes more evident in the fourth, fifth and sixth trials (figs. $4 \mathrm{D}, 4 \mathrm{E}, 4 \mathrm{~F}$ ), when epicarp lesions rarely formed. To illustrate the change in damage levels during the time when shell hardening is completed, we compared the average number of old epicarp lesions (those that formed 2 to 4 weeks after inoculation) across inoculation periods and showed a significant reduction between the June 8 and 22 inoculation periods for redshouldered stink bug, flat green stink bug and leaffooted bug (fig. 5).

Levels of kernel necrosis were low in the May and early-June inoculation treatments, with levels increasing in later treatments to a peak in late July, followed by a slight decrease in August (fig. 6). We suggest that the lower lev- 


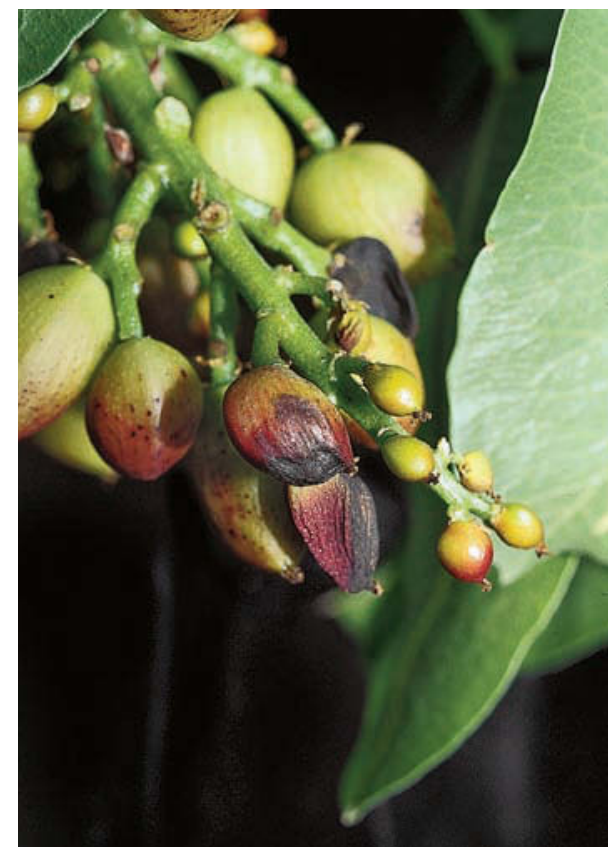

The number of surface lesions to still-soft pistachio shells can be a good tool for monitoring insect feeding activity early in the season.

els found in May and June, when the shell hardening is not yet complete, is the result of an increase in aborted nuts (data not provided). Throughout all inoculation periods, adult leaffooted bugs caused more damage than the adult stink bugs tested. As suggested by Rice et al. (1985) and Michailides et al. (1987), it is clear that adult leaffooted bugs can feed on the kernel throughout the season. Still, these bugs were caged for 7 days on a single cluster and damage levels were less than $20 \%$, and we suspect that the hardened shell significantly reduces insect feeding.

\section{Practical applications}

We showed that in the early-season period, before crop load is set, insectdamaged nuts are dropped from the cluster; due to the plant's natural compensation for these dropped nuts, such insect feeding will not result in crop loss in most years and orchards. Significant crop loss will occur only when early-season bug densities are so high that the number of dropped nuts exceeds the level for which the tree will naturally compensate. For this reason, from mid-April through May the developing nuts should be monitored for insect damage, and the pistachio canopy and orchard floor should be monitored for small and large bugs.

The midseason period, as the pistachio shell is hardening and the fruit load is set, is the most critical period since the large bug mouthparts can easily penetrate the shell and most of the damaged nuts remain in the cluster. During this period, not all damaged nuts will have the externally visible epicarp lesions, and some lesions may require up to 2 weeks to develop. Therefore, the damaged kernel may not be apparent until harvest, when nuts are processed and graded.

In the late-season period when the shell has hardened, bug damage was significantly reduced in our studies (but see Michailides et al. 1987). We suggest that mid- and late-season insect sampling should focus on large bugs in the pistachio canopy, especially the adult leaffooted bugs, which are most capable of penetrating the hard pistachio shell. Therefore, it is helpful to properly identify small and large bugs and monitor their corresponding damage.

Accurately sampling bug densities is difficult. Because reliable sampling methods and economic action thresholds that correlate to economic crop loss have not yet been developed, managers should use a variety of sampling tools (sweep nets, beating trays and visual inspection) to keep abreast of changes in insect densities. In addition, the number of epicarp lesions can be a good tool for monitoring insect feeding activity early in the season, when lesions may form within 48 hours of a wound. However, be more cautious when monitoring lesions during the midseason period, since there can be a 2-week period between the initiation of insect feeding and the development of lesions. After shell hardening is complete, insect-damaged nuts will not be readily visible and when found will indicate feeding that probably occurred weeks or even months before. Therefore, insecticide applications that target epicarp lesions may be applied long after the insect damage occurred. For this reason, if bugs are found in the orchard after shell hardening, the best way to assess crop damage is to inspect the kernel for signs of insect damage.
K.M. Daane is Associate UC Cooperative Extension (UCCE) Specialist, and G.Y. Yokota, R. Krugner, and S.A. Steffan were Staff Research Associates, Division of Insect Biology, UC Berkeley (currently, Krugner is Graduate Student, UC Riverside, and Steffan is Project Manager, Utah State University); P.G. da Silva was Postgraduate Student, Division of Insect Biology, UC Berkeley (and currently is Associate Professor, College of Marin); R.H. Beede is Farm Advisor, UCCE Kings County; W.J. Bentley is Areawide Farm Advisor, UC Statewide IPM Program, UC Kearney Agricultural Center; and G.B. Weinberger is owner, Weinberger $\mathcal{E}$ Associates, Visalia. We thank the California Pistachio Commission, UC Statewide IPM Program and Western Region SARE program for funding; and Louise Ferguson, UCCE Specialist, Department of Pomology, UC Davis, for technical advice with pomology horticulture. Farm managers at Sherman Thomas Ranch, Nichols Farm, Agri-World and Paramount Farms provided field sites. Comments from two anonymous reviewers greatly improved the manuscript.

\section{References}

Abbott WS. 1925. A method for computing the effectiveness of an insecticide. J Econ Entomol 18:265-7.

Beede RH, Rice RE, Daane KM, et al. 1996. Relation of Phytocori relativus to lecanium scale infestations on pistachios. In: California Pistachio Industry Annual Report, Crop Year 1995-96. California Pistachio Commission, Fresno, CA. p 107-18.

Bolkan HA, Ogawa JM, Rice RE, et al. 1984. Leaffooted bug implicated in pistachio epicarp lesion. Cal Ag 38(3-4):16-7.

Bostock RM, Thomas CS, Ogawa JM, et al. 1987. Relationship of wound-induced peroxidase activity to epicarp lesion development in maturing pistachio fruit. Phytopathol 77:275-82.

Michailides TJ, Ogawa JM, Rice RE. 1988. Sites of epicarp lesions and kernel necrosis in relationship to symptoms and phenology of pistachio fruit. J Econ Entomol 81:1152-4.

Michailides TJ, Rice RE, Ogawa JM. 1987. Succession and significance of several hemipterans attacking a pistachio orchard. J Econ Entomol 80:398-406.

Purcell M, Welter SC. 1991. Effect of Calocoris norvegicus (Hemiptera: Miridae) on pistachio yields. J Econ Entomol 84:114-9.

Rice RE, Uyemoto JK, Ogawa JM, Pemberton WM. 1985. New findings on pistachio problems. Cal Ag 39(1):15-8.

Uyemoto JK, Ogawa JM, Rice RE, et al. 1986. Role of several true bugs (Hemiptera) on incidence and seasonal development of pistachio fruit epicarp lesion disorder. J Econ Entomol 79:395-9. 